The discovery of a $58 \mathrm{keV}$ line feature in the spectrum of the X-ray source HerX-1 by J. Trümper and his colleagues from Tübingen \& Garching (Germany) in $1976{ }^{1}$ ), as reported in Europhysics News, 8 (1977) 5, is the latest exciting news from the rapidly expanding field of X-ray astronomy. Cosmic X-rays are completely absorbed by the earth's atmosphere, and therefore the observing instruments have to be placed in the outer layers or outside the atmosphere. Up to 1970 , balloon and rocket flights had led to the discovery of about 30 X-ray sources. But then, in December 1970, NASA launched the Uhuru satellite which was devoted entirely to X-ray astronomy. This was the big step forward, because within less than 2 years, Uhuru had found about 100 galactic and about 50 extragalactic X-ray sources. About $90 \%$ of these galactic sources apparently constituted a new class of stellar objects with an X-ray luminosity exceeding the sun's total luminosity by a factor of $10^{5}$. This was substantiated by satellite observations in later years.

The new satellites also revealed new types of galactic X-ray stars, so that now one knows of a large variety of types, of which regular pulsating sources, and sources showing irregular fluctuations (as e.g. Cyg X-1 on a timescale of milliseconds) appear less exotic than the flare-like objects, which appear, reach a peak luminosity, and then fade away as if they had never been. But most peculiar are the burst-like sources, which emit their X-rays in short term bursts of a few seconds duration, with no apparent periodicity involved. The theorists do not hesitate, however, to invoke equally exotic images, when they try to explain the observations. Although we are far from understanding these $\mathrm{X}$-ray stars completely, we have learned quite a bit during the past years. Some general, fairly obvious, but not necessarily compelling conclusions (there is always a more complicated fairy tale with the same outcome) can be drawn immediately from the data:

The rapid variability on timescales of seconds or milliseconds (cf. Table 1) suggests that a compact object (white dwarf, neutron star, black hole) is involved. The energy source for the $\mathrm{X}$-rays comes from gas which accretes in the deep gravitational well of the compact object, flowing over from a companion star, which is not compact. This view is supported by the fact that for 10 X-ray sources, it has already been established that they are members of a binary system (several are listed in Table 1), i.e. they are in orbit around an optical companion. As long as there is no evidence to the contrary, we may live happily with the assumption that all other galactic $\mathrm{X}$-ray sources are also binary systems with mass flow, and we may wait confidently for further candidates to Table 1.

Most of the energy is liberated deep inside the gravitational well, i.e. on the stellar surface, if the compact object is a neutron star, or within a few Schwarzschild radii, if the object is a black hole. In both cases, for 1 solar mass objects, distances are $\cong 10^{6} \mathrm{~cm}$. A proton in free fall on to a $1 M_{0}$ object would have a kinetic energy of $\cong 100 \mathrm{MeV}$ at such a distance. If one thermally radiates away the energy in $\mathrm{X}$-rays of a few keV, i.e. at temperatures of $T \cong 10^{7}$ to $3 \times 10^{7} \mathrm{~K}$, from areas with such typical dimensions, one finds luminosities of $10^{36}$ $10^{38} \mathrm{erg} / \mathrm{s}$.

This is in good agreement with the observations. Talking only of black holes and neutron stars is, of course, unfair to the white dwarfs, which can also satisfy the constraints imposed by the data. But accretion on white

Table 1

\begin{tabular}{|c|c|c|c|c|c|}
\hline Source & Short term variability & Long term variability & Optical candidate & $\begin{array}{l}\text { Distance } \\
(\mathrm{kpc})\end{array}$ & $\begin{array}{l}\text { Luminosity }(2-10 \mathrm{keV}) \\
\left(10^{36} \mathrm{erg}\right)\end{array}$ \\
\hline $\begin{array}{l}\text { HerX-1 } \\
(3 \cup 1653+35)\end{array}$ & $1.24 \mathrm{~s}$ pulsations & $\begin{array}{l}\text { X-ray eclipses (1.7d) } \\
35 d \text { modulation } \\
\text { long-term on-off }\end{array}$ & $\begin{array}{l}\mathrm{HZ} \text { Her ; } \\
1.7 \mathrm{~d} \text { binary }\end{array}$ & $2-6$ & $1-10$ (6 kpc) \\
\hline $\begin{array}{l}\text { Cen X-3 } \\
(3 \cup 1118-60)\end{array}$ & 4.84 pulsations & $\begin{array}{l}\text { X-ray eclipses ( } 2.1 d) \\
\text { extended lows }\end{array}$ & $\begin{array}{l}\text { double ellipsoidal } \\
\text { light variation } \\
\text { Ob star }\end{array}$ & $6-9$ & $10-30(9)$ \\
\hline $\begin{array}{l}\text { Cyg X-1 } \\
(3 \cup 1956+35)\end{array}$ & $\begin{array}{l}\text { Irreg. var. in times } \\
\text { of milliseconds }\end{array}$ & $\begin{array}{l}\text { slow transition } \\
\text { between } 2 \text { distinct } \\
\text { spectral states }\end{array}$ & $\begin{array}{l}\text { HDE } 226868 \\
5.6 d \text { binary }\end{array}$ & 2.5 & $3-10$ var \\
\hline $\begin{array}{l}\text { Vela X-1 } \\
(3 \cup 0900-40)\end{array}$ & $\begin{array}{l}283 \text { s pulsations } \\
(282.8913 \pm 0.0004)\end{array}$ & $\begin{array}{l}\text { flares, eclipse } \\
\text { (8.95d) }\end{array}$ & HD 77581 & 2 & 10 \\
\hline $\begin{array}{l}\text { SMCX-1 } \\
(3 \cup 0115-73)\end{array}$ & $0.71 \mathrm{~s}$ pulsation & $\begin{array}{l}\text { X-ray eclipses, } 3.9 \mathrm{~d} \\
\text { extended lows }\end{array}$ & $\begin{array}{l}\text { SK } 160 \\
13 \text { mag. }\end{array}$ & 60 & $30-300$ \\
\hline $\begin{array}{l}\text { ScoX-1 } \\
(3 \cup 1617-15)\end{array}$ & Irreg. var. min & $\begin{array}{l}\text { slow ( } ~ 10 \text { min-h) } \\
\text { flares }\end{array}$ & $\begin{array}{l}\text { ScoX-1, blue } \\
0.787 \mathrm{~d} \text { period }\end{array}$ & $1-2$ & 10 \\
\hline A0620-00 & none & $\begin{array}{l}\text { appears in outbursts } \\
\text { lasting } \approx 1 \text { year } \\
(1967 \& 1975)\end{array}$ & $\begin{array}{l}\text { optical } \\
\text { identification } \\
\text { no period } \\
\text { determined }\end{array}$ & 1 & 100 \\
\hline
\end{tabular}



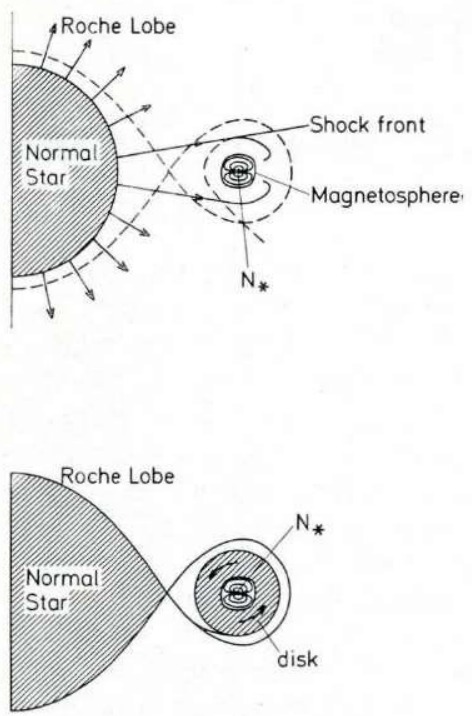

Fig. 1. - Schematic drawing of accretion from a stellar wind and Roche Lobe overflow with disk accretion.

dwarfs produces keV X-rays only, if the infall is radial; the observed period changes are very difficult to explain, and strong magnetic fields, as measured by Trümper '), cannot be supported by white dwarfs. With accretion on to neutron stars or black holes, about $10 \%$ of the rest mass energy of the infalling gas is converted into X-rays. Thus the accretion rates do not have to be very large: $10^{-8} \mathrm{M}_{0} / \mathrm{a}$ to $10^{-10} \mathrm{M}_{0} / \mathrm{a}$ is sufficient to give the powers observed.

These or larger mass-transfer rates can easily occur in close binary systems. Three different mechanisms may be important :

i) A massive companion expanding across the critical equipotential surface between the two stars - Roche lobe overflow - could transfer mass at a rate of $10^{-6}$ $\mathrm{M}_{0} / \mathrm{a}$ to $10^{-3} \mathrm{M}_{0} / \mathrm{a}$.

ii) A stellar wind, i.e. an atmosphere streaming radially outwards, as expected from $\mathrm{Ob}$ or Be stars, can have a magnitude of $10^{-6} \mathrm{M}_{0} / \mathrm{a}$. The accretion rate on to the orbiting neutron star or black hole would then be about $10^{-9} \mathrm{M}_{0} / \mathrm{a}$.

iii) A feedback mechanism, where the heating of the companion by $\mathrm{X}$ rays, produces the mass flow necessary to substain the X-rays, may also be possible. Such a mechanism may be applicable to the HerX-1 system.

It is probable that because of the angular momentum of the accreting gas, the mass flow will lead to the formation of an extended flat disk around the compact object (the disk will probably be smaller in the case of a stellar wind). The physics of the accretion disk determines many fea- tures of the X-ray source (in Fig. 1, a schematic drawing of the gas flow is presented). Table 1 (ref. 2) contains several regularly pulsating $\mathrm{X}$-ray sources, with pulse periods in the range of $1.24 \mathrm{~s}$ (HerX-1) - $283 \mathrm{~s}$ (Vela $X-1)$. The time-keeping mechanism is probably rotation of the compact object, which means rotation of a neutron star at least for the fast rotators. The observed pulses must be produced by an asymmetry in the infall of matter, and the obvious way to achieve this is to have inclined to the axis of rotation, a stellar magnetic field, which guides the matter down, such that only certain hot spots on the surface accrete. As these hot spots move through our field of vision, we see X-ray pulses.

\section{Measurement of the Magnetic Field of a Neutron Star}

What information do we gain from observations on the physics of the accretion process close to a neutron star? The spectrum of the radiation emerges from a complicated and intricate interaction of many distinct features, such as hot plasmas accelerated by a strong gravitational field, guided by a rotating magnetic field, finally hitting a neutron star surface of very high density. There are so many parameters unknown, that a quantitative picture of the accretion process is not available, one only knows that it must be quite complicated.

There are great hopes, however, for an improvement of the theoretical situation, due to the measurement by the group of J. Trümper. They have observed the regularly pulsating source HerX-1 for about 4 hours in a balloon flight from Palestine/Texas on May 3, 1976. The spectrum for the pulsed flux they have obtained is shown in Fig. 2. There is strong evidence of a spectral line feature at 58 $\pm 5 \mathrm{keV}$, which has an intensity of $3.10^{-3} \mathrm{~cm}^{-2} \mathrm{~s}^{-1}$, that is several percent of the total pulsed flux. The best estimate of the line width is $\leq 12 \mathrm{keV}$. The most likely explanation for this line seems to be in terms of an electron cyclotron emission, because atomic or nuclear emission mechanisms fail to produce the high intensity required. If the line emission is due to the radiation of electrons spiralling around the magnetic field lines of the star, then the line frequency is a direct measure of the magnetic field strength. $58 \mathrm{keV}$ corresponds to a magnetic field of $B=5.10^{8} \mathrm{~T}$. Such a high value of the magnetic field can only be expected for a neutron star. This experiment establishes therefore that HerX-1 is a neutron star. It also establishes that neutron stars really have the large magnetic fields expected for them from theoretical considerations of stellar collapse, and from the slowing down of the pulsars by the emission of electromagnetic dipole radiation. Meanwhile a second experiment by Trümper et al. has confirmed the results of the first measurements, and other observers have also obtained evidence for a line feature in the spectrum of HerX-1.

The large value of $5.10^{8} \mathrm{~T}$ also indicates that the line emission is a quantum effect. In strong magnetic fields, the motion of the electron perpendicular to the field is quantized, similar to the quantization of the electron orbits in an atom. The energy difference between two adjacent orbits or levels is $\hbar \omega_{B}$ where $\omega_{B}=$ $e B / m c$ is the classical gyration frequency. A spin reversal of the electrons has the same energy difference $2 \pi h \omega_{B}$. So there are two excited states with the same energy difference with respect to a ground state : a higher orbit quantum number, or a different spin direction of the electron on the same orbit. Transitions into the ground state from the first excited state in this system of Landau levels produce the observed line. The emission of higher harmonics should also be possible. There is, indeed, an indication of an enhancement in the observed spectrum around $110 \mathrm{keV}$, where one would expect the second harmonic to occur.

Fig. 2. - Measurement of J. Trümper et $\left.a l^{1}\right)$ showing the spectrum of the HerX-1 pulses and of OSO-8 (Aug. 75) showing the total spectrum.

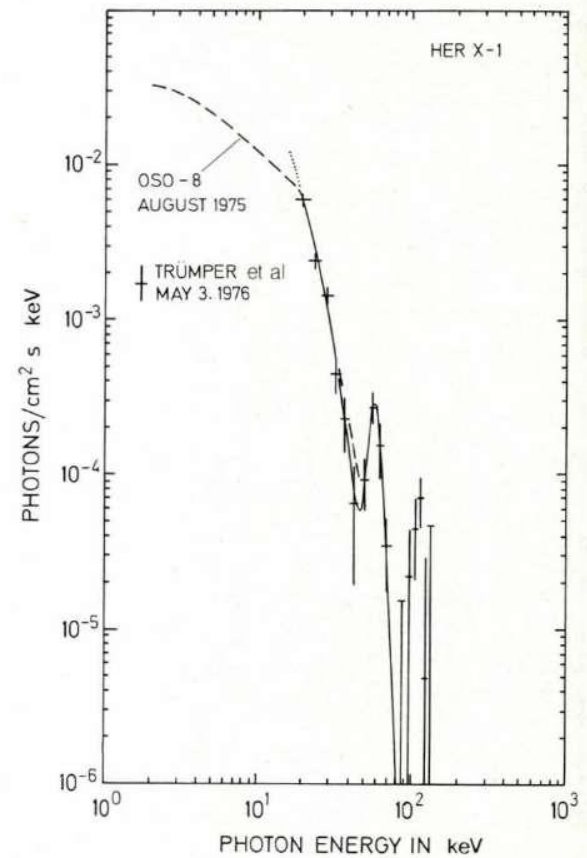


The pulsed continuum radiation above $25 \mathrm{keV}$ can be fitted by an exponential spectrum of $\cong 8 \mathrm{keV}$ cutoff, and the $58 \mathrm{keV}$ cyclotron line is sitting on top of it, assuming it is seen in emission. It is likely that the hot plasma emitting the $X$-rays is optically thin with respect to the continuum radiation (the free-free absorption coefficient is much smaller than the Thomson scattering coefficient). The absorption coefficient at the cyclotron frequency is, in contrast, much larger and the black body intensity in the emission line should be reached causing the line to stand out above the continuum. It is thus not a contradiction to assume an electron temperature in the accretion channel right above the neutron star surface, greatly in excess of the average $k T$ of $8 \mathrm{keV}$. In fact $k T_{\mathrm{c}}$ should be greater than $\sim 40-50 \mathrm{keV}$, to populate the higher Landau levels sufficiently and excite the line. It is difficult to understand how the protons transfer several percent of their kinetic energy to the electrons. Coulomb collisions and collective plasma excitations may be important. It is only fair to say that the excitation mechanism for the line is not understood at present.

On the other hand, one can already draw some conclusions from the observed line width. If the main broading mechanisms are Doppler broadening and self-absorption, both depending on the angle of the line of sight of the observer with the magnetic field, the small width enforces the conclusion that the cyclotron line is seen from a direction close to perpendicular to the fields, i.e. the radiation must show a fan-like pattern. The same mechanism is probably responsible for the appearance of pulses in the other spectral regions, since putse shapo, phase and duty cycle are similar in the continuum and the line. This fan-beam is of course very plausible, because along the accretion columns the photons are dragged downwards, making it much easier for the radiation to escape sideways than upwards.

There are potentially great rewards in this observation, rewards for the theorist, but more work is required to arrive at a quantitative model for the formation of the pulses. The flow pattern of the plasma in the neutron star's magnetic field, i.e. the structure of the accretion column, determines the opacities in the various directions, the structure of the hot spot, and therefore also the region where the line originates. The plasma flow in turn is influenced by the interaction of the disk of incoming gas with the neutron star's magnetic field. The field

starts to dominate around a radius of $1000 \mathrm{~km}$, and the $X$-rays come from a region near the neutron star surface with a radius of $10 \mathrm{~km}$. The whole region of this extended magnetosphere affects the formation of the $X$-ray pulses. In that sense, the line measurements provide an invaluable diagnostic tool in determining details of the accretion mechanism on to a neutron star.

\section{Mass Determination of Neutron Stars in Binary Systems}

Besides the observations of spectra and pulse shapes, and hence a value for the magnetic field of a neutron star, the existence of these $X$-ray souices in binary systems, allows the determination of their masses within rather narrow limits. For regularly pulsating sources such as HerX-1, one can, from the Doppler shift of the $X$-ray pulses, obtain radial velocities and finally a value for the mass function $\left(M_{p}^{3} \sin ^{3} i\right) /\left(M_{x}+M_{0}\right)^{2}$, which depends only on the mass of the optical companion $M_{p}$, on the angle $i$ between the normal to the orbital plane and the line of sight, and on the mass of the $X$-ray source $M_{x}$. In several cases (Table 2) the optical companion has been identified, and its mass can be derived from the spectral type within certain limits. Then one needs information on the angle of inclination from other observations, to determine $M_{\mathrm{x}}$.

The source HerX-1 shows periodic occultations with a period of $1.7 \mathrm{~d}$. The optical companion, $\mathrm{Hz} \mathrm{Her}$, exhibits regular variations by two magnitudes in phase with the $X$-ray eclipses. This means that the light variations are caused by irradiation from the $X$-ray source. For the mass function one finds a value of $0.85 \mathrm{M}_{0}$. The spectral type (late $A$ to $F$ ) gives - together with $\sin i=1$, which seems plausible from theoretical considerations of the 35 day cycle of this source: $M_{1}=1.7-2.2 M_{0}$ and $M_{p}=0.7-1.4 M_{1}$ and a distance of $5 \mathrm{kpc}, \mathrm{cf}^{2}$ ).

For sources, where the irradiation by X-rays of the optical companion is negligible, one can find sinusoidal variations in the optical light curve with twice the orbital period. These are due to an ellipsoidal deformation of the star by the X-ray source. As

\section{Table 2}

Source

HerX-1

CenX-3

SMCX-1

Vela $X-1$

Cyg X-1

$M_{\mathrm{x}} / M$,
$\cong 1$
$0 . \overline{\underline{6}}-1.8$
$2.2-4.2$
$1.3-2.2$
$8.5-15$

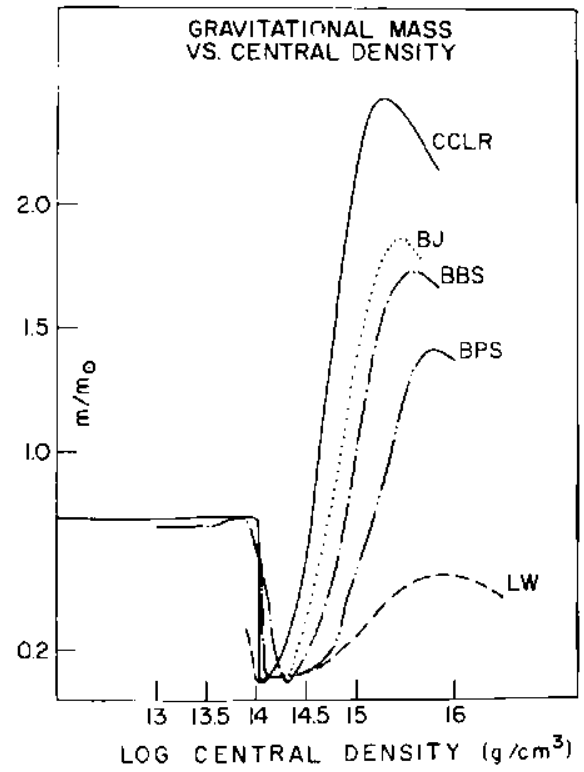

Fig. 3. - Masses of neutron stars for various realistic equations of state derived from a 2-body potential using many-body techniques; taken from ref. 5 , where complete references can be found.

the X-ray source orbits the star, we see the ellipsoid head on (minimum light) - sideways (maximum) - head on - sideways during one revolution.

A precise analysis of these light curves is quite involved, but it clearly depends very strongly on the mass ratio of the two components ${ }^{2}$ ).

From Doppler shifts of spectral lines in the optical, one can determine $c$ mass function $\left(M_{x}^{3} \sin ^{3} i\right) /\left(M_{1}+M_{x}\right)^{2}$ even for nonpulsating $X$-ray sources. The regularly pulsating source, Vela $X-1$, allows a determination of an optical as well as an X-ray Doppler shift, and thus the mass value for this source is determined very accurately.

The results of this light curve analysis (Avni \& Bahcall ${ }^{2}$ ), are listed in Table 2. One can see that neutron star masses (i.e. masses of regularly pulsating $X$-ray sources) are within the theoretical limits of $0.2-2.5 \mathrm{M}_{0}$. It is interesting to note that the masses seem to be rather high $\left(\supseteq 1 M_{t}\right)$ a fact which may be relevant with regard to the evolutionary history of these objects.

\section{Black Hole in Orbit}

In Table 2 we find a minimum mass $M_{\mathrm{x}}=8.5 M_{\mathrm{i}}$ for Cyg X-1. This innocent looking fact has made this object one of the most widely discussed in recent years.

White dwaits have masses less than $1.4 \mathrm{M}_{0}$, and also the masses of neutron star models do not seem to exceed a maximum of $2.5-3 \mathrm{M}_{\mathrm{n}}$ (cf. Fig. 3). These models have been calculated with many-body methods from 


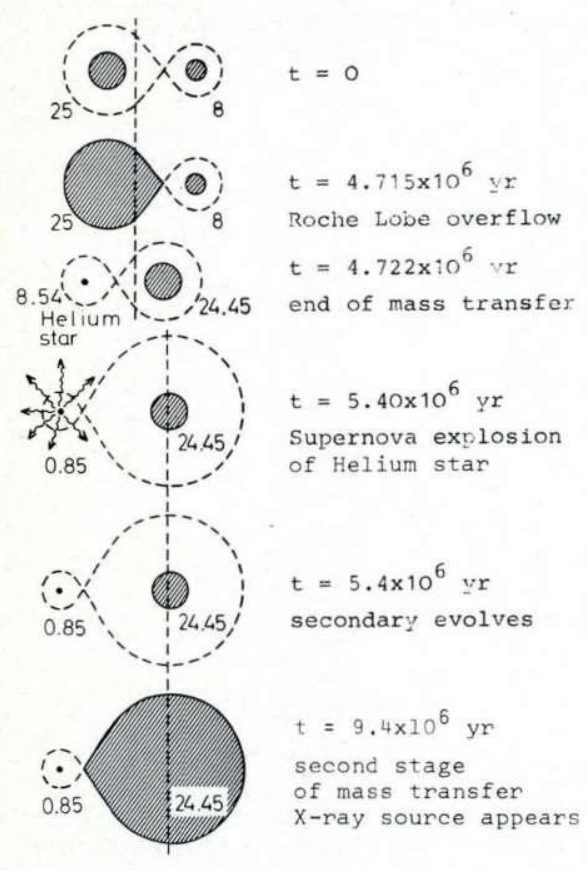

Fig. 4. - A possible evolutionary sequence for a binary system ${ }^{3}$ ).

a 2-body potential which is attractive at large separation and becomes repulsive at short distances. But large extrapolations from the density regime accessible by laboratory experiments with nuclei, had to be made in the derivation of the precise shape, especially of the repulsive part of the 2-body potential. So the reliability of this upper mass limit for neutron stars could be questioned. The Cyg X-1 mass determination instigated theoretical investigations into absolute upper mass limits using only assumptions such as positive density, positive pressure-density gradient, but no specific equations of state for densities above normal nuclear density. In addition, these limits were investigated in Einstein's theory of general relativity as well as in other more exotic theories of gravitation. When astrophysicists indulge in such free speculations, the result is to be expected: no absolute upper mass limit seems to exist. But for the more conservative mind, content to stay within the limits of Einstein's theory, an absolute upper mass limit of $5-8 M_{0}$ seems to exist. This then has the consequence that $\mathrm{Cyg}$ $\mathrm{X}-1$ is the first established representative of the sinister class of black holes, collapsed objects which hide behind an event horizon, through which no radiation can escape. Only when they accrete matter they become visible through the radiation of the hot plasma which surrounds them.

The radiation from Cyg $\mathrm{X}-1$ has peculiar characteristics. Short-term fluctuations occur on a time-scale of milliseconds, indicating an extension of the source of $\leq 100 \mathrm{~km}$. The average spectrum changes between two distinct states of low and high intensity for the soft X-rays between 2 and $6 \mathrm{keV}$. These are puzzling properties, but so far they are not understood. It is also not clear whether the accretion on to a black hole has characteristic features which distinguish it from other accretion processes. Therefore the evidence for a black hole in orbit rests on the somewhat indirect argument about its mass exceeding the mass limit of other compact objects.

\section{Evolution}

How do neutron stars in binary systems come into being? For the massive systems with a massive blue supergiant as the companion, an evolution along the lines of Fig. 4 may have occurred (after ref. 3).

After the primary (the more massive, and therefore more rapidly evolving) of initially $25 \mathrm{M}_{0}$, say, has burnt its hydrogen core, contraction and hydrogen shell burning sets in, during which phase the hydrogen envelope of the primary is almost completely transferred to the secondary (of initially lower mass, say $8 \mathrm{M}_{0}$, and therefore less rapidly evolving). The primary is then a helium star with helium core burning, and $M \leq 4 \mathrm{M}_{0}$. Its lifetime after the onset of oxygen burning is much less than the thermal timescale of the envelope (neutrino emission cooling).

Then the core collapses before the star reaches its critical Roche lobe. This collapse and supernova explosion, will not disrupt the system, because the secondary is still unevolved, and has a much larger mass. The remnant is a black hole or a neutron star, which may for some time appear as a pulsar.

The secondary then evolves away from the main sequence, to a blue supergiant, produces a strong stellar wind, and by accretion on to the compact object, the X-ray source appears.

There are some difficulties with this general picture. It is still unknown, under what conditions supernova ex- plosions leave a neutron star as a remnant. One possible mechanism would be the collapse (electron capture) of an iron core above the Chandrasekhar limit. But an iron core would require a rather massive initial configuration. Smaller mass stars seem to get completely disrupted in a $\mathrm{SN}$ explosion, because of explosive $\mathrm{C}^{12}$ burning. So the evolution of systems such as $\mathrm{HZ}$ Her-Her $\mathrm{X}-1$, which contain only $3 \mathrm{M}_{0}$, is ill understood. Model calculations also indicated that it is difficult to obtain the correct binary periods.

As the secondary evolves further it will eventually overflow the Roche lobe, and cover the X-ray source. It may then be possible that the compact source spirals inward inside the secondary, blowing a lot of mass out of the system, until finally it orbits a white dwarf like core. The core may undergo a supernova explosion too, leaving a binary system of two neutron stars, or a neutron star and a black hole. This could be the story of the birth of a binary pulsar as PSR 1913 +16 .

\section{Binary Pulsar}

The pulsar PSR $1913+16$ has been discovered in 1975 by Hulse \& Taylor ${ }^{4}$ ) (parameters in Table 3 ). It is in a very narrow binary system : (projected along the line of sight, the periastron is $R_{0} \approx 7 \times 10^{10} \mathrm{~cm}$ ). A normal star cannot fit in, therefore the pulsar's companion must be a small object, either a compact object or a helium dwarf. If the companion really is a compact object, then this system is a unique laboratory for tests of general relativity. A precise clock (the pulsar period) in orbit presents the opportunity of measuring relativistic effects such as periastron advance, spin precession, effects of gravitational radiation on the orbital parameters.

The radial Doppler shifts have been measured and the mass function has been obtained. The periastron advance has recently been found to amount to $4 \%$ a. If this is due only to relativity, one knows the sum of the masses to be $2.8 \mathrm{M}_{0}$. Another measurement, e.g. of the spin precession, would determine all system parameters. Any further measurement of

\section{Table 3 Binary Pulsar PSR1913+16}

Period

Flux at $430 \mathrm{MHz}$

Eccentricity of orbit

Longitude of periastron

Rate of advance of periastron

Projected semimajor axis

Mass function
$0.0590301 \pm 0.0000002 \mathrm{~s}$

$0.006 \pm 0.003$ Jansky

0.6

$179^{\circ}$

$4 \%$

a. $\sin i=1.00 \pm 0.02$ solar radii

$f(m)=0.13 \pm(0.01) \mathrm{M}_{\circ}$ 
a relativistic effect, would then also be a test of Einstein's theory of gravitation.

\section{Conclusion}

It is fascinating to see how, starting with the discovery of the pulsars, many more and ever more exotic astronomical objects involving neutron stars have been found. These massive bodies of densities inaccessible in terrestrial laboratories, seem to be set up in such a variety of celestial systems that their properties can be unravelled step by step. These are good times for astrophysicists.

\section{References}

1. TRÜMPER, J., PIETSCH, W., REPPIN, C., VOGES, W., STAUBERT, R., and KENDZIORRA, E., Ap. J. Letters 1978 (in press)

2. "X-Ray Binaries" Proc. of NASA Symp. at GSFC (Maryland) (1975). This volume has been used as a standard reference for the data presented.

3. VAN DEN HEUVEL, E.P.J. and HEISE, J. Nat. Phys. Sci. 239, 1972, 67

4. TAYLOR, J.H., HULSE, R.H., FOWLER, L.A., GULLAHORN, G.E., and RANKIN, J.M., Ap. J. 206, 1976, L53

5. BÖRNER, G., Springer Tracts in Mod. Phys. 69 (1973) 1

\section{Universiteit van Amsterdam}

The group for Atomic Physics in the Zeeman Laboratorium has a vacancy for a

\section{senior research associate $(\mathrm{m} / \mathrm{f})$}

who will participate in current activities (term analysis of spectra and laser spectroscopy), with the special duty to take charge of theoretical support. Applicants should be qualified specialists in the field of theoretical atomic structure and spectroscopy and have a good knowledge of experimental methods. Educational duties include the teaching of theory of atomic spectroscopy at an advanced level and coaching of graduate students. Command of the English language is mandatory while a reasonable knowledge of Dutch is expected to be acquired during service. Applications with curriculum vitae, list of publications and names of referees to be received before July Ist, by

Dr. P. F A. Klinkenberg Zeeman Laboratorium Plantage Muidergracht 4 IOI8 TV Amsterdam - The Netherlands.

\section{Lattice Dynamics}

An International Conference on Lattice Dynamics was held in Paris, September 5-9, 1977, sponsored by EPS, IUPAP and SFP. The programme of this conference reflected recent developments in this field, a large part being devoted to phase transitions related to mode softening and central peak. The remarkable developments of non-linear physics have also penetrated the field of lattice dynamics : a session was devoted to solitions. Much interest seems to focus on phonon driven phenomena: superconductivity, ferroelectricity, melting and to non perfectly (ill) condensed matter, defects in materials, disordered phases, amorphous solids and liquids and materials of specific interest like superionic conductors.

Instead of an overall account of the Conference a few significant contributions in the fields of Microscopic Theory, Solitons and Electron-Phonon Interactions are summarized.

\section{Microscopic and Model Theories of Lattice Dynamics}

Microscopic calculations of phonon dispersion curves are much simpler in metals than in non-metals. The po- tential energy of electrons, in simple metals at least, is small compared to their kinetic energy: the ratio can then serve as a small parameter. The electronic response to core displacements can be described in terms of a simple complete set of equilibrium functions and yield a unified formulation of microscopic lattice dynamics both in metals and non-metals. A rigorous "first-principle" calculation has yet to be performed. The effort in this direction is nevertheless interesting for at least two reasons: microscopic theory should help us understand in detail how the dynamical spectrum of solids arises from their crystal and electronic structures employing Fermi surface effects, covalent, ionic, metallic binding, etc. The second reason is that such theory should connect the same macroscopic physics to related properties such as superconductivity, crystallographic phase transitions, optical and transport properties and the like.

S.K. Sinha 1) advocates the approach using the density response function of the electron system. With the formulation using this approach, he examines the occurrence of phonon soft-

\section{Balkanski, Paris}

(Chairman, Condensed Matter Division)

ening and lattice instabilities in d-band metals pointing out a strong correlation between such behaviour and a high density of states at the Fermi level associated with the d-states, and also with the occurrence of relatively high $T_{c}$ in superconductivity. Model calculations considering several quasi-degenerate d-bands at the Fermi level yields, for $\mathrm{Nb}$, the characteristic dips in the longitudinal branches along [100] and [111]. The dip along [111] is probably related to the "central peak" observed in $\mathrm{Nb}$ and $\mathrm{Nb}-\mathrm{Zr}$ alloys. $\mathrm{NbC}$ and $\mathrm{TaC}$ show also pronounced softening type anomalies in the acoustic modes reproduced well by the same model. It has also been pointed out that the response function $\mathrm{X}_{\mathrm{c}}(\vec{q})$, peaks in $\mathrm{NbC}$ due to Fermi surface nesting features, which occur also at the position of the anomalies. This may indicate correlation between the electron-phonon matrix elements and the electronic structure.

A similar coincidence with regard to peaks in $X_{\mathrm{c}}(\vec{q})$ and the position in $q$ of the maximum softening, is mentioned in the "charge-density-wave" materials, e.g. the layered transition 